\title{
It's Still Simple: Signal Plus Response Equals Communication
}

\author{
Thomas C. Baker
}

Published online: 9 April 2014

(C) Springer Science+Business Media New York 2014

I was fortunate to have been in on the ground floor of insect pheromone research "back in the day" when only a handful of pheromone structures had been identified. During these ensuing 42 years, I was privileged to have been able to witness many advances in our understanding of insect pheromone communication systems. Yet, I have been perplexed by the continued inattention of researchers, both young and old, to the concept of "communication". With the many advances in analytical techniques that have occurred over these decades, it has become easier to isolate and chemically identify many more compounds from pheromone glands or airborne volatile collections than in earlier times. But it also has become easier to make the mistake of assuming that all of the identified compounds contribute to the behavioral response, i.e., that they all comprise the signal between the sexes that evokes the optimal behavioral response.

Much of what contributes to this mistake is a lack of attention to the simple idea that communication involves a "signal" and a "response". The response that has been selected for over evolutionary time is a behavioral one, not an EAG, not a de-orphanized odorant receptor gene, and certainly not whatever bunch of compounds elutes off a GC/MS. Rather, the response is the whole insect behaviorally being attracted to the emitter of the signal and then mating (or trying to mate) with that sender. Additionally, with regard to the signal, it is not the entire emission blend that a researcher is capable of collecting and chemically characterizing from a gland or an airborne volatile collection device, but rather the minimal set of compounds in that collection that can be proven, as a blend, to contribute to behavior so as to fully evoke attraction to the source as effectively as the natural extract or the pheromone-emitting moth itself.

In my early days in the field, we were well aware of the importance of distilling down the possible set of behaviorally active sex pheromone components to its minimum, because it was important not only to achieve the simplest, most economically formulated blend for commercial monitoring lures or for mating disruption, but also because it was crucial to understanding the evolution of these signals. If a research group has a lot of behaviorally inert side-products that they are calling pheromone components, any ideas they put forth about selection pressures that may have shaped that pheromone system will be erroneous because they will have mistakenly labored to take into account variations in behaviorally meaningless compounds that have not been evolutionarily selected for at all. If a person does not understand what is a behaviorally inert biosynthetic byproduct and what is the real communication signal, then wild stories can be dreamed up that will have nothing to do with reality, with evolution. Nowadays, this inattention to diligently using discriminating behavioral assays to narrow down complex emissions to a minimal set (i.e., the signal) that induces optimal behavior is more likely to occur in the many

T. C. Baker $(\bowtie)$

Distinguished Professor of Entomology and Chemical Ecology,

Pennsylvania State University, University Park, PA, USA

e-mail: tcb10@psu.edu studies involving inducible defenses in plants and their effects on natural enemies and other herbivores. This is because host-plant volatile emissions have so many compounds, are highly complex, and vary so much from plant to plant, cultivar to cultivar, and yet without knowing the true signal among all those compounds, how can we figure out how it shifts over time to keep the plant protected?

Today, I see another type of inattention to behavioral responses, and this is with regard to truly understanding that the response to a pheromone by a flying insect is a visual one, involving variations in the wind-induced optomotor response to image motion around the insect. I can remember when the landmark advance in understanding behavioral responses to pheromones occurred early-on in pheromone research. This was the eye-opening paper by Kennedy and Marsh (1974) that surprised us all with the revelation that male moths do not steer according to a chemical pheromone gradient in order to arrive at the pheromone emitter, but rather, they steer with respect to the wind by using visual image-motion feedback from the environment. The concept that a pheromone merely switches-on a wind-steering program and does not itself chemically guide the male in any way was revolutionary and left us shaking our heads in wonder at the ingenious way the Kennedy group was able to demonstrate this, by rotating a floor pattern beneath a pheromone-stimulated male flying in a wind tunnel. For some reason (perhaps because we ourselves do not fly), this important behavioral concept, and a behavioral reality, keeps getting ignored or at least incompletely understood by folks young and old in the field, decade after decade.

It is especially important right now to truly understand this intimate link between olfaction and vision due to the great progress that has been made in neuroanatomical studies of olfactory pathways far up into the protocerebrum. I do hope that today's researchers can fully grasp that the response to pheromone (and other volatiles) by flying insects is nearly entirely a visual response to wind, and not to the pheromone itself.

If visual stimuli are not included in stimulus panels when olfaction researchers record from these higher-order neurons, then a key opportunity will have been lost to help give us new clues as to how visual imageflow might synergize or suppress the responses to pheromone blends by multimodal visual-plus-olfactory neurons in the protocerebrum.

What J. S. Kennedy's group opened our eyes to 40 years ago during the inaugural year of publication of the Journal of Chemical Ecology should always be kept in mind: there is an intimate, and mandatory, coupling of olfaction with vision during odor-mediated flight by insects. Ignorance of this fact can easily lead to missed opportunities for discovery. Or worse, it may result in an off-course foray into many of the promising, lovely-scented headwinds in chemical ecology research.

\section{References}

Kennedy JS, Marsh D (1974) Pheromone-regulated anemotaxis in flying moths. Science 184:999-1001 